\title{
Ann Arbor Stage II Supradiaphragmatic Hodgkin Lymphoma
}

National Cancer Institute

\section{Source}

National Cancer Institute. Ann Arbor Stage II Supradiaphragmatic Hodgkin Lymphoma.

NCl Thesaurus. Code C5009.

Ann Arbor Classification: Stage II: Involvement of two or more lymph node regions on the superior side of the diaphragm (II) or localized involvement of a single associated extralymphatic organ or site and its regional lymph node(s) with or without involvement of other lymph node regions on the same side of the diaphragm (IIE). Note: The number of lymph node regions involved may be indicated by a subscript. 